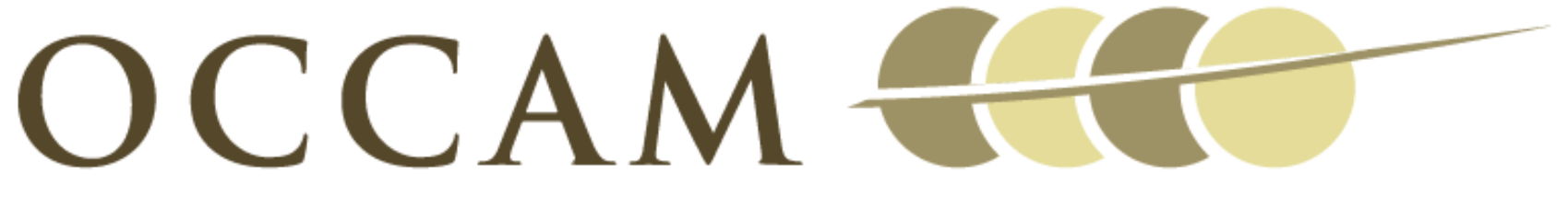

OXFORD CENTRE FOR COLLABORATIVE APPLIED MATHEMATICS

Report Number 11/66

On the shape of force-free field lines in the solar corona

$$
\text { by }
$$

C. Prior and M.A Berger

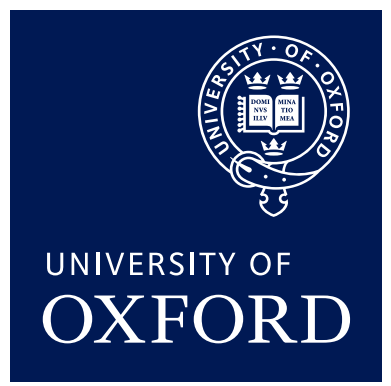

Oxford Centre for Collaborative Applied Mathematics Mathematical Institute

24 - 29 St Giles'

Oxford

OX1 3LB

England 



\title{
On the shape of force-free field lines in the solar corona
}

\author{
C. Prior $^{1}$ - M.A. Berger ${ }^{2}$
}

(c) Springer

\begin{abstract}
This paper studies the shape parameters of looped field lines in a linear force-free magnetic field. Loop structures with a sufficient amount of kinking are generally seen to form $S$ or inverse $S(Z)$ shapes in the corona (as viewed in projection). For a single field line, we can ask how much the field line is kinked (as measured by the writhe), and how much neighbouring flux twists about the line (as measured by the twist number). The magnetic helicity of a flux element surrounding the field line can be decomposed into these two quantities. We find that the twist helicity contribution dominates the writhe helicity contribution, for field lines of significant aspect ratio, even when their structure is highly kinked.

These calculations shed light on some popular assumptions of the field. First, we show that the writhe of field lines of significant aspect ratio (the apex height divided by the foot point width) can sometimes be of opposite sign to the helicity. Secondly, we demonstrate the possibility of field line structures which could be interpreted as $Z$-shaped, but which have a helicity value sign expected of an $S$-shaped structure. These results suggest that caution should be exercised in using two dimensional images to draw conclusions on the helicity value of field lines and flux tubes.
\end{abstract}

Keywords: Corona, Structures;Helicity, Magnetic, Observations;Magnetic fields, Corona

\section{Introduction}

Observations of the corona reveal the existence of S-shaped features, the signature one would expect of a helical rope viewed from above. These structures were termed sigmoids by Rust and Kumar (1996), and are generally seen to occur in both forward and reverse $\mathrm{S}$ shapes (termed $\mathrm{Z}$ sigmoids by Low and

\footnotetext{
${ }^{1}$ Oxford Centre for Collaborative Applied Mathematics (OCCAM), Mathematical Institute 2429 St Giles' Oxford OX1 3LB email: priorc@maths.ox.ac.uk

${ }^{2}$ Mathematics, CEMPS, University of Exeter, North Park Road, Exeter EX4 4QF, United Kingdom email: m.berger@exeter.ac.uk
} 
(a)

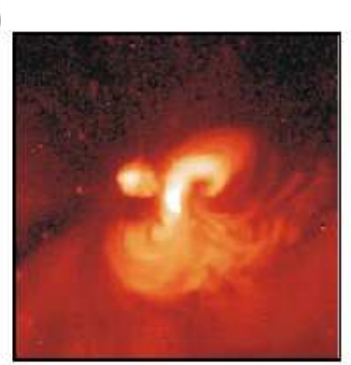

(b)

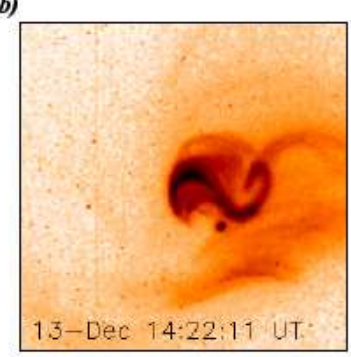

Figure 1. Figure (a) shows a sigmoid before an eruption. It was observed on 8 June 1998 15:19 UT, by the Yohkoh Soft X-Ray Telescope. Figure (b) is an example of a reverse S sigmoid from the Yohkoh Soft X-Ray Telescope. Both are reprinted from the Montana state university solar physics department website (solar.physics.montana.edu).

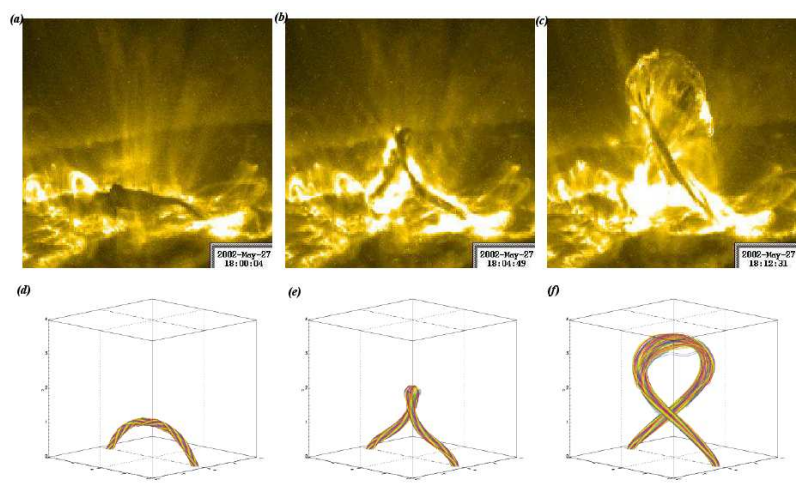

Figure 2. The top row of figures are images of a confined filament eruption on 2002 May 27 , shown in extreme ultraviolet light by the TRACE satellite. The right image shows the filament after it has reached its maximum height. The bottom figures represent the evolution of a kink-unstable flux rope from a simulation performed by (Török and Kliem, 2005). From left to right the flux rope starts as a tube shape which lies in a single plane. Then we see the rope structure contort in the $x-y$ plane to form a helical structure

Berger (2003)); examples are shown in Figure 1. They may result from enhanced dissipation from an accumulation of hot plasma along correspondingly shaped field lines (Green et al., 2007). Nakagawa et al. (1971) provided an explanation of the origin of such structures by describing the magnetic fields in sun spot regions as twisted flux ropes. The suggestion was that the handedness of the sigmoids was related to the sense of twist in the magnetic field. For a field with right handed twist, i.e. positive chirality, the central portion of a sigmoid would rotate in a clockwise direction during its evolution; left handed twist would lead to an anticlockwise rotation (negative chirality).

Deriving information on the helicity of coronal field structures from observational data is a difficult task, much of the obtained observational data does not necessarily provide sufficient information for knowledge of field line's full three-dimensional structure. TRACE imaging (Handy and et al., 1999) provides 


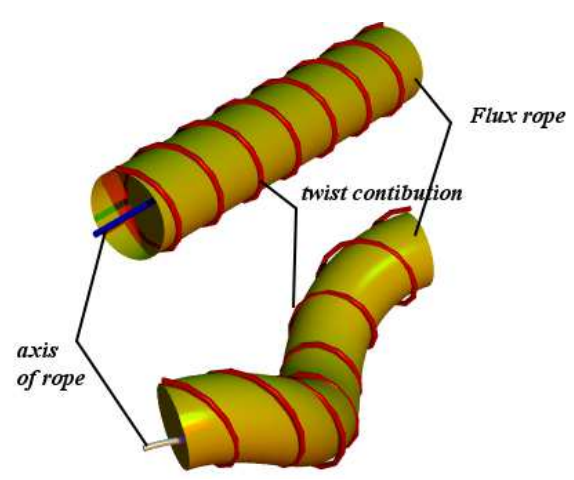

Figure 3. Two flux ropes are depicted. The curves on their surfaces represent twisted field lines. The axis of flux rope (a) is a straight line. Thus the shape of its axis will have zero contribution to the tube's helicity. The helicity of the tube is entirely determined by the degree of its twisting. As the axis of flux rope (b) is helical, it has a non-zero writhe helicity contribution.

line of sight imaging of magnetic structures in the corona. The Yohkoh satellite (Tsuneta and et al., 1991) and its follow up mission Hinode (Kosugi and et al., 2007) are used used to follow the evolution of structures in the corona, also using two-dimensional imaging. The STEREO satellite mission employs two identical observatory satellites, one ahead of the earth's orbit and one behind. The aim is to provide stereoscopic measurements of the corona (Kaiser, 2005). The STEREO mission may be able to offer a more accurate picture of the threedimensional nature of magnetic coronal structures than the two dimensional methods.

A flux rope can be thought of as a set of magnetic field lines which bunch together to create a twisted flux bundle with a mutual axis. Flux ropes in closed magnetic regions often exhibit helical structure (see for example Figure 2). This particular morphology is generally thought to occur as a result of the kink instability, for which a straight cylindrically twisted flux rope becomes unstable to perturbation at a critical twisting value. The kink instability is conjectured to play a role in observable phenomena such as coronal brightenings and coronal mass ejections (Titov and Demoulin, 1999; Magara and Longcope, 2003; Török and Kliem, 2005).

If a flux rope is closed (i.e. it has no discernible endpoints) its helicity splits into two components: the writhe $\mathcal{W}$, measuring the kinking of the rope's central axis, and the twist $\mathcal{T}$, measuring how much neighbouring field lines wind about the axis (illustrated in Figure 3). For a flux tube with axial flux $\Phi$, the helicity is

$$
H=\Phi^{2}(\mathcal{T}+\mathcal{W})
$$

(Berger and Field, 1984). The sum of these components is a topological invariant which represents the extent to which these two curves link each other. This measure is known as the linking number $(\mathcal{L})$, thus $H=\Phi^{2} \mathcal{L}$, where $\mathcal{L}=\mathcal{T}+$ $\mathcal{W}$. The last equation is the well known Călugăreanu theorem (Călugăreanu , 


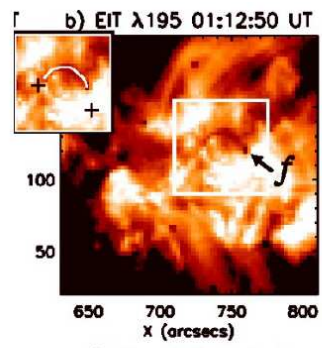

f) Simulation: $t=0$

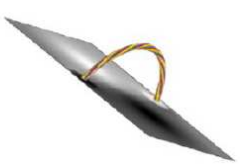

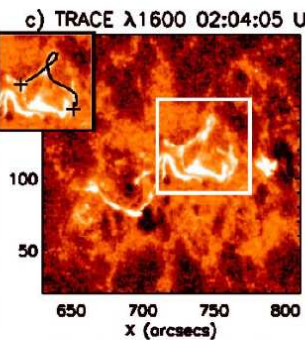

g) Simulation: $t=21 \tau_{\mathrm{A}}$

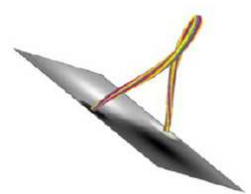

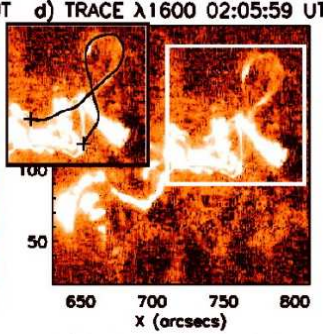

h) Simulation: $t=25 \tau_{A}$

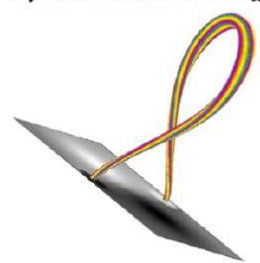

Figure 4. Figures b, c, and d depict a filament eruption and coronal mass ejection on October 10th 2004 in the NOAA active region 10696. The images are TRACE 1600 images. Figures f-h are from an MHD simulation performed by Török and Kliem (Török, Kliem, and Titov, 2004). The times marked on the figures are Alfvén times. Figures b-d would appear to suggest a shape which is not fully symmetric (about its maximum height (apex)). This figure is reprinted from Green et al.(Green et al., 2007).

1959;1961). This particular decomposition has often been discussed in literature concerning coronal flux ropes (Rust, 2003; Fan and Gibson, 2004; Tian et al., 2005; Green et al., 2007; Pevstov, 2008).

In cases of open-ended field lines, or flux tubes bounded by a surface such as the photosphere, Berger and Field (1984) showed that the helicity can be defined by subtracting the helicity of a reference field (i.e. a potential field), which has the same normal component at the boundary. Using this definition for the helicity, Berger and Prior (2006) have derived expressions for the $\mathcal{T}$ and $\mathcal{W}$ which apply to flux ropes with footpoints on a boundary plane. The writhe expression was called polar writhe to distinguish it from quantities used in the DNA literature. The polar writhe measure was recently used by Török, Berger, and Kliem (2010) to evaluate the (polar) writhe helicity of MHD simulations of active region flux ropes.

In the corona, helicity can be shown to be conserved to an excellent approximation (Heyvaerts and Priest, 1984; Berger, 1984). Thus, the increasing writhing of the tube's axis must be compensated for by a change in twisting. We use the term change with care here. If the twist and writhe components are of opposing sign, an increase in the magnitude of the writhing would lead to an increase in the magnitude of the twisting. If they are of the same sign an increase in writhing magnitude would lead to a reduction in the magnitude of twisting. Clearly the specific type of kinking which occurs could have an effect on the flux tube's behaviour. In this note we demonstrate cases for which the tube's helicity is positive and the flux rope has significant negative writhing, leading to an increase in twisting (sections 4 and 5 ). 
This paper will illustrate the decomposition of helicity into twist and writhe using the field lines of a linear force-free field. Such fields have been used as a starting configuration for several models of flux ropes in active regions. Previous studies concerning the morphology of flux ropes applied to cases with a high degree of symmetry (Titov and Demoulin, 1999; Low and Berger, 2003; Fan and Gibson, 2004; Török and Kliem, 2005); these simulations have tended to develop symmetrical sigmoidal structures. Specifically, the field lines can be split into two halves at a centre-point; each half can be obtained from the other by rotating thorough $180^{\circ}$ in the $x-y$ plane. For these geometries the height of the loop is the dominant factor controlling the sign of the writhe (Berger and Prior, 2006; Török, Berger, and Kliem, 2010). It is not clear from observational data however that such symmetry is the norm (see Figure 4). Further, the effect of asymmetry on the writhing (and helicity) of helical field line configurations has not yet been examined in detail.

We pay particular attention to two common assumptions regarding first the helicity and secondly the writhing of magnetic flux ropes.

- $\mathrm{S}$ shapes are generally associated with flux ropes of positive helicity and $\mathrm{Z}$ shapes negative helicity, consistent with the observed hemispheric chirality laws (Rust and Kumar, 1996; Green et al., 2007). Some exceptions may be noted. Low and Berger (2003) describe flux rope field models where the height of a field line dips near the middle of the line. For such lines, one can obtain the opposite relation between $\mathrm{S}-\mathrm{Z}$ shape and chirality. Such cases have been found in simulations (Magara and Longcope, 2003; Magara and Longcope, 2001; Kliem, Titov, and Török, 2004).

- For field lines of significant aspect ratio (the ratio of height to foot-point width), which trace out forward $\mathrm{S}$ shapes, the writhe will be negative, if the aspect ratio is greater than $h \approx 0.4$. This assumption is made by Green et al. (2007), based on the results of symmetric helical forms detailed in Berger and Prior (2006), see also Török, Berger, and Kliem (2010). For Z shaped sigmoids this relationship is reversed. As an example of this relative scale, Török and Kliem (2005) simulate a confined flux tube eruption, indicative of an active region, whose aspect ratio ranges from 1-4.

The results of the force free calculations presented here include counter examples to both of these assumptions. First, we find cases of field lines in a positive helicity field with a $\mathrm{Z}$ shaped projection, but without a central dip. For the second assumption we find both positive and negative writhe values for $S$ shaped field lines. Thus care must be taken in inferring the sign of writhe and helicity from observing an $\mathrm{S}$ or $\mathrm{Z}$ shape; some consideration must be given to both asymmetries in the field line structure as well as the height of the structure and the presence or absence of dips.

It must be stressed that these results are not used to comment on any particular flux rope or active region field models. The simple linear force free model used does not have the sophistication of the MHD simulations used to model coronal eruptions. Nor are they used to contradict any current theories. The aim of this exercise is to demonstrate the variety of three-dimensional geometries which display helical structure. Many studies have been based on the plethora of two 

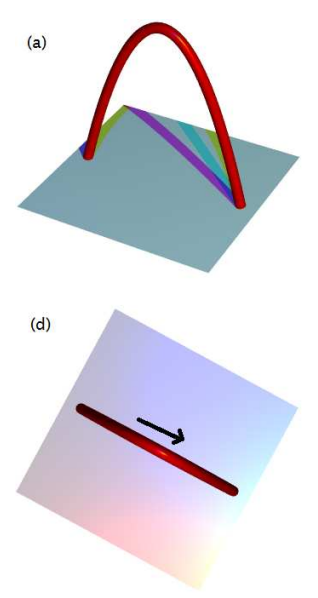

(b)

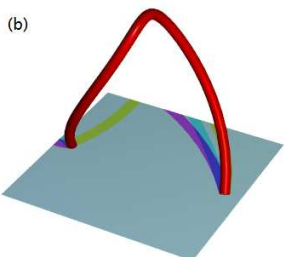

(e)

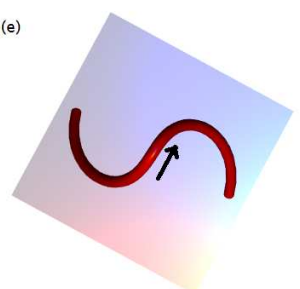

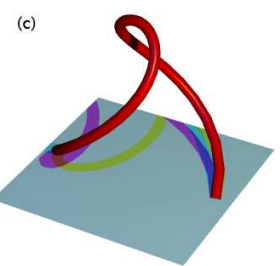

$(f)$

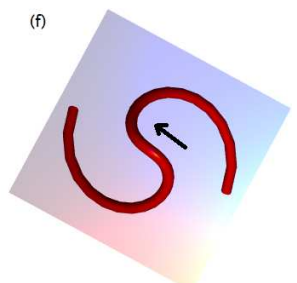

Figure 5. Several helical flux tube shapes with increasing amounts of kinking. We see three helical shapes, labelled (a), (b), and (c); figures (d), (e), and (f) are respectively their projections onto a plane as viewed from above. As the kinking increases the central section of the tube rotates in an anti-clockwise direction.

dimensional images currently available. For example Green et al. (2007); Liu et al. (2008); and Muglach, Wang, and Kliem (2009), have provided evidence for specific active region field models, based on the observation of the rotation of sigmoid structures using soft x-ray imaging. These studies imply that the rotation of the central portion of this field is an indication of the conversion of twisting, of the flux rope, into writhing of its axis (see Figure 5).

However, the writhe has two contributions, termed local and nonlocal in Berger and Prior (2006). The nonlocal writhe measures how much the two legs of a loop twist about each other, and can be read off of two-dimensional projections. The local writhe, however, measures local contortions of the axis, and requires three-dimensional information. The local writhe can have an important effect on determining the writhing and twisting of the flux rope. In general the two writhe contributions are of opposing sign leading to writhe values which are smaller than one might expect upon viewing the structure.

The paper will be structured in the following manner.

In Section 2 we discuss the general geometrical properties of coronal flux ropes. Section 3 details the simple force free models we use to generate field lines. We examine both a simple two component Fourier model (Section 4) and a more realistic quadrupolar field (section 5), both with positive helicity.

For each field we describe numerical evaluations of the writhe of field lines for various start positions and $\alpha$ values. We choose to focus on high $\alpha$ values as they produce field lines of high aspect ratio. Attention is then paid to the morphology of these high $\alpha$ field lines. This is done for a range of starting points which often encompass several separatrix surface boundaries. We find that the writhing takes on both positive and negative values based on the relative magnitudes of the local and non-local writhing components. Their helicity is always positive and 

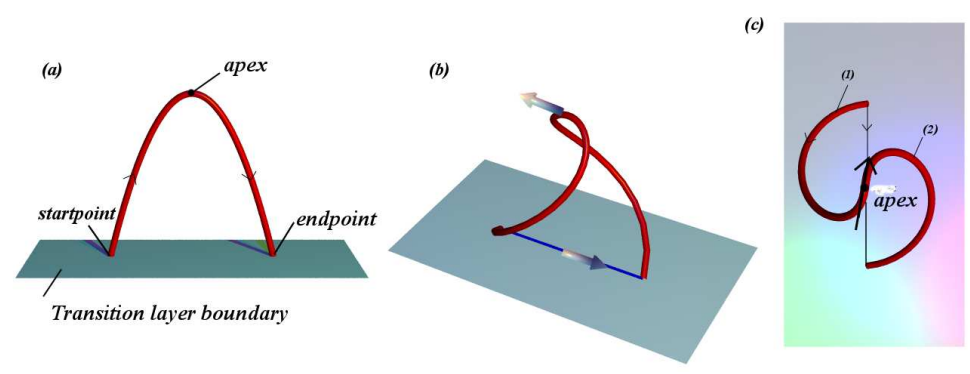

Figure 6. Diagrams (a) to (c) are representative of the type of field lines morphologies present in magnetic flux rope sigmoid models. (a) is an example of a field with no writhe, it draws out a parabolic shape in the $y-z$ plane. Plot (b) is an example of a field line structure which has a degree of writhing. Arrows mark the orientations between the foot-points and the orientation at the apex point. Plot (c) represents the field in (b) as viewed from above.

generally dominated by the twist component. The sign of the writhing is shown to be, in part, a function of the particular behavioural mode to which its belongs. The quadrupole fields are shown to generate field lines which appear to have a $\mathrm{Z}$ shaped morphology, at least in their central region, but with only one local maximum (no dip). These structures have positive helicity values which match their $\alpha$ value.

We summarize and conclude in section Section 6 .

\section{The flux rope helicity expression}

\subsection{Characteristics of a sigmoidal field line}

The simplest linear force free field lines have one maximum, forming a loop in the solar atmosphere (Priest, 1981 section 3.5.2), see (a) in Figure 6. The start and end foot-points of the field line will lie in the $z=0$ plane. The apex represents the point on the curve of maximum field line height (see (a) in Figure 6). In what follows all curves generated will have only one maximum in $z$, a global maximum. Finally, when we discuss the aspect ratio, it shall be understood to be the apex height divided by the foot-point width.

Field lines often form helical type structures (Nakagawa et al., 1971; Gary, 1989), see (b) and (c) in Figure 6. Projecting these field lines onto the $x-y$ plane can be thought of as equivalent to viewing the field from above. We note in (c) (Figure 6) the loop forms an $\mathrm{S}$ shape under this projection. The sigmoidal shape in Figure 6 has a degree of symmetry about the curve's apex. One half of the curve can be rotated, using the apex as a pivot, either clockwise or anticlockwise through an angle of $\pi$ radians, in order to superimpose it onto the other half. When we talk about symmetry and asymmetry in what follows it is with respect to this rotation. Perfect implies a perfect overlap. Asymmetry is not specifically characterized any further than an imperfect overlap and will be referred to in a qualitative manner. 


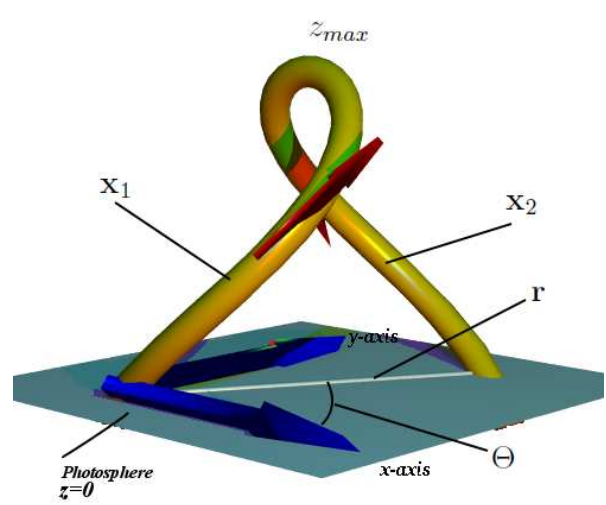

Figure 7. A helical loop structure. The loop has footpoints on the photospheric boundary $z=0$. It has a maximum height $z_{\max }$. The loop can be split into two sections by its maximum height; these sections are labelled $\mathbf{x}_{1}$ and $\mathbf{x}_{2}$. The vector $\mathbf{r}(0)$ joining these two curves is shown. Its orientation with the $x$-axis $\Theta$ is also shown.

\subsection{The polar writhe}

We consider a field line $\mathbf{x}(s)$, expressed in Cartesian co-ordinates and parametrized by its arc length $s$. This curve represents the axis of a flux tube which is fully contained in a region between two planes $z=0$ (the photospheric surface) and $z=z_{\max }$. We define the polar writhe $\mathcal{W}_{p}$ of $\mathbf{x}$ as

$$
\mathcal{W}_{p}\left(\mathbf{x}, 0, z_{\max }\right)=\mathcal{W}_{p l}\left(\mathbf{x}, 0, z_{\max }\right)+\mathcal{W}_{p n l}\left(\mathbf{x}, 0, z_{\max }\right)
$$

Here $\mathcal{W}_{p l}$ and $\mathcal{W}_{p n l}$ represent the local and non-local components of the polar writhe (Berger and Prior, 2006; Török, Berger, and Kliem, 2010). We can split the curve into two sections, $\mathbf{x}_{1}$ and $\mathbf{x}_{2}$, via its apex, as depicted in Figure 7 . We first consider the local component. The field line's local geometry can be determined by analysing the unit tangent vector $\widehat{\mathbf{T}}$. The local writhe component is expressed in terms of the rate of rotation of $\widehat{\mathbf{T}}$ about the $z$ axis.

In the case of our helical structures $\mathcal{W}_{p l}$ will always take the following form

$$
\mathcal{W}_{p l}\left(\mathbf{x}, 0, z_{\max }\right)=\frac{1}{2 \pi} \int_{0}^{z_{\max }}\left(\frac{\hat{z} \cdot\left(\widehat{\mathbf{T}}_{1} \times \widehat{\mathbf{T}}_{1}^{\prime}\right)}{1+\left|\hat{z} \cdot \widehat{\mathbf{T}}_{1}\right|}+\frac{\hat{z} \cdot\left(\widehat{\mathbf{T}}_{2} \times \widehat{\mathbf{T}}_{2}^{\prime}\right)}{1+\left|\hat{z} \cdot \widehat{\mathbf{T}}_{2}\right|}\right) \mathrm{d} z
$$

where $\widehat{\mathbf{T}}_{i}^{\prime}$ are derivatives of $\widehat{\mathbf{T}}_{i}$ with respect to arc length. In order to evaluate the non-local writhing of helical field line structures we define a vector $\mathbf{r}(z)$, $\mathbf{r}(z)=\mathbf{x}_{2}(z)-\mathbf{x}_{1}(z)$, which lies in the $x-y$ plane. This vector joins the two points on the field lines with the same height $z$; its orientation with the $x$-axis is denoted $\Theta(z)$ (see Figure 7). We can evaluate the non-local writhing in terms of the orientations of the field line's apex point and the orientation of its footpoints. If we define the orientation of the field line at its apex as $\Theta_{\max }$ and the orientation of the foot-points in the $x-y$ plane as $\Theta_{\text {min }}$, then $\mathcal{W}_{\text {pnl }}$ can be 
expressed as

$$
\mathcal{W}_{p n l}\left(\mathbf{x}, 0, z_{\max }\right)=\frac{1}{\pi}\left(\Theta_{\min }-\Theta_{\max }\right)+2 n,
$$

where $n$ represents the number of full rotations of the vector $\mathbf{r}$ between $z=0$ and $z=z_{\max }$.

\subsection{Observing the writhe}

For helical structures which have no dip it is possible to observe the non-local writhing using two-dimensional projection. This can be achieved by evaluating the differing orientations of the foot-points and the apex of the sigmoid, as observed in either $\mathrm{H} \alpha$ or soft x-ray imaging (Liu et al., 2008). This method does not take into account any full windings ( $n$ in Equation (4) of the field structure; though, as discussed in Berger and Prior (2006), this number will, in most cases, be zero. Observing the changing rotation of the apex section would allow one to track the changing value of the non-local writhing helicity of sigmoidal brightenings, for example Green et al. (2007); Liu et al. (2008); and Muglach, Wang, and Kliem (2009) perform such observations.

The local writhing is another matter. In order to quantify the local writhing accurately we must be able to specify the full three-dimensional structure of the tube's axis. This is beyond the scope of the two-dimensional imaging techniques discussed in the introduction. As we shall see in Section 4, the local writhing component, for field lines of significant aspect ratio, is often of a similar magnitude to that of the non-local winding, so this limitation is significant.

\subsection{The twist helicity}

We shall be using a linear force-free field model described below, where $\nabla \times \mathbf{B}=$ $\alpha \mathbf{B}$, in order to generate the axis of the fields. The flux tubes of linear force-free fields have a constant twisting rate about their axis. This rate is characterized by a constant $\alpha$. Thus the net twist will be

$$
\mathcal{T}=\frac{\alpha s}{4 \pi}
$$

(Berger and Prior, 2006), where $s$ is the arc length of the tube's axis $\mathbf{x}$.

\subsection{Flux tube helicity}

Using Equation (1) we can now define the helicity of our flux tube as

$$
H\left(0, z_{\max }\right)=\Phi^{2}\left(\mathcal{W}_{p}\left(\mathbf{x}, 0, z_{\max }\right)+\frac{\alpha s}{4 \pi}\right)
$$

In what follows we set $\Phi$ to unity for all helicity evaluations, with no loss of generality. 


\section{A simple force free model}

\subsection{Linear force free fields}

We now define a simple linear force free field model in order to generate asymmetric field line structures. The various regions of the sun's atmosphere are generally modelled as an ideal electrically neutral plasma (Priest, 1981). If the plasma is in equilibrium then the plasma's motion can be considered to be zero and the field can be described by the magneto-hydrostatic equation and its subsidiary equations,

$$
\begin{array}{r}
0=-\nabla p+\mathbf{j} \times \mathbf{B}+\eta \mathbf{g}, \\
\mathbf{j}=\nabla \times \mathbf{B} / \mu, \\
\nabla \cdot \mathbf{B}=0,
\end{array}
$$

where $\mathbf{B}$ is the magnetic field and $\mathbf{g}$ the gravitational force. The scalar quantities $p, \eta$ and $\mu$ are the plasma pressure, magnetic diffusivity, and the magnetic permeability respectively. From left to right the three contributions to the R.H.S of (7) come from the plasma pressure gradient, the Lorentz force, and the gravitational force. In the corona, the field structures can be stable on time-scales of hours to days (Gary, 1989).

Further, in the corona the plasma pressure and gravitational force are small relative to the magnetic pressure (Priest, 1981); as a result of this they may be ignored for equilibrium fields and the Lorentz force will tend to dominate in this region. Using these assumptions (7) reduces to the force free field equation

$$
\nabla \times \mathbf{B}=\alpha \mathbf{B} .
$$

The factor $\alpha$ may vary spatially but is fixed along field lines. A simple form of (10) has $\alpha$ as a constant for the volume of interest; this is termed the linear force-free model. Using the linear model, the field can be reconstructed above $z=0$, for a specified $\alpha$ value. In the following work we are considering theoretical configurations of the coronal field, generated by the linear force-free model, and will be specifying the magnetic field entirely as an initial value problem.

\subsection{The model}

A divergence-less field can be split into scalar poloidal $(P)$ and toroidal $(T)$ components (Morse and Feshbach, 1953):

$$
\mathbf{B}(x, y, z)=\mathbf{L} \cdot T(x, y, z)+\nabla \times \mathbf{L} \cdot P(x, y, z),
$$

where $\mathbf{L}=\hat{z} \times \nabla$ is the angular momentum operator. We will separate the solution (B) of (10) in this fashion. Solutions can be obtained by defining the poloidal term as a Fourier expansion (Mackay et al., 1997)

$$
\begin{aligned}
P & =\sum_{k \neq 0} \frac{1}{k^{2}} B_{k} \mathrm{e}^{\mathrm{i} \mathbf{k} \cdot \mathbf{x}_{p}} \mathrm{e}^{-\left(k^{2}-\alpha^{2}\right)^{1 / 2} z}, \\
T & =\alpha P .
\end{aligned}
$$




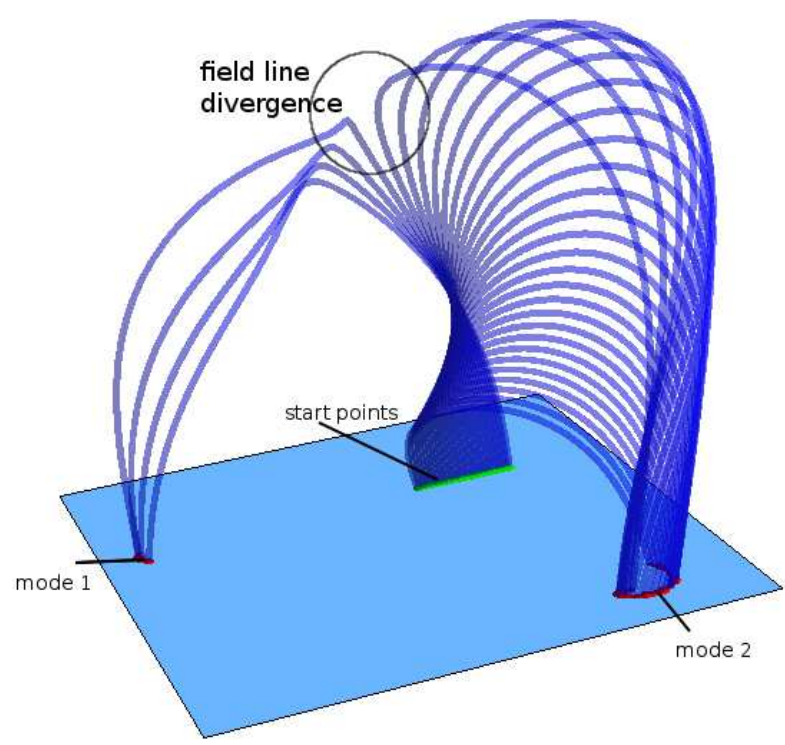

Figure 8. A depiction of two different behavioural modes of $\mathbf{B}$. The tubes are field lines which are generated by our Runge-Kutta solver. The green dots represent their start points and the red dots their end points. There are two distinct behavioural modes, as defined by the two different lines of end points, which are labelled mode 1 and mode 2. Separatrix points represent the points on the boundary at which the divergence occurs.

where

$$
\mathbf{k}=k_{x} \hat{x}+k_{y} \hat{y}, \quad \mathbf{x}_{p}=x \hat{x}+y \hat{y}, \quad k^{2}=k_{x}^{2}+k_{y}^{2},
$$

and $B_{k}$ are the Fourier coefficients. If we require that the field components are bounded then the factor $\sqrt{k^{2}-\alpha^{2}}$ must be real (Gary, 1989); thus imposing a limit on the possible range of $\alpha$ values. Using this representation the field is

$$
\mathbf{B}(x, y, z)=\sum_{k \neq 0} \alpha\left(\nabla_{H} P\right) \times \hat{z}-\beta\left(\nabla_{H} P\right)+k^{2} P \hat{z} .
$$

Where

$$
\nabla_{H}=\frac{\partial}{\partial x} \hat{x}+\frac{\partial}{\partial y} \hat{y} .
$$

In particular by specifying $B_{z}$ at the photosphere $(z=0)$ we can define the whole field as an initial value problem. In what follows we define a specific form for $B_{z}(x, y, z=0)$ as a Fourier series. The value of $\alpha$ is then specified, based on the Fourier components present in the specified $B_{z}$ form, and the field line is reconstructed using a second order Runge-Kutta algorithm.

\subsection{Separatrix layers}

The basic definition of a separatrix surface is a boundary separating two modes of behaviour of a differential equation. The notion of separatrix boundaries in coro- 
(a)

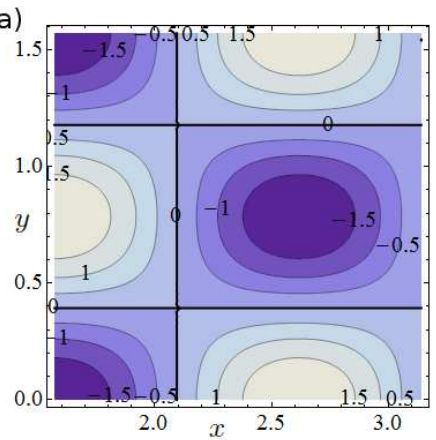

(b)

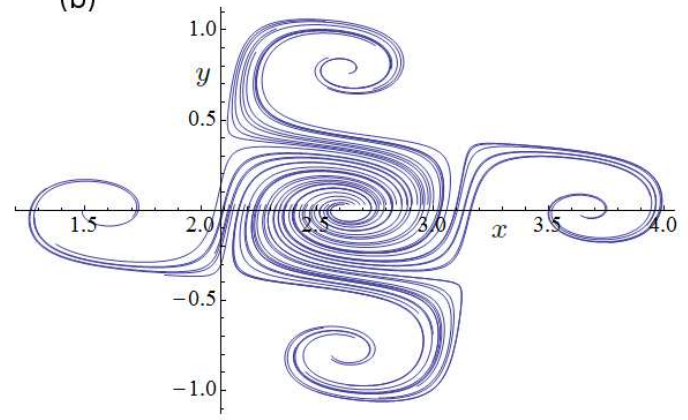

Figure 9. Figure (a) is a contour plot depicting the $B_{z}$ component of the field used in Section 4 to generate field lines. The domain shown is $x \in[\pi / 2, \pi] y \in[0, \pi / 2]$. Density values are shown on the plot and the polarity inversion lines are shown as a bold lines. In (b) we depict projections onto the $x-y$ plane of the $S$-shaped field lines produced by this $B_{z}$ distribution. The lines represent an equal sampling of the domain $x \in[2.1,3]$ and $y=0.01 \pi$, whose characteristic helicity measures are shown in Figure 10.

nal field models is discussed in some detail in Longcope (2005). In this note the field lines are three dimensional structures and the notion of differing behaviour is in the divergence of field lines which have neighbouring start points, but whose end points differ significantly on the plane $z=0$ (see Figure 8). In sections 4 and 5 we shall be varying the start position along sets of the $x-y$ plane which represent lines. As the solutions start and end at the photospheric layer $z=0$, we can think of the generation of the field line as a map $M\left(x_{s}, y_{s}\right) \rightarrow\left(x_{e}, y_{e}\right)$, from the start footpoint $\left(x_{s}, y_{s}\right)$ to the end footpoint $\left(x_{e}, y_{e}\right)$. As depicted in Figure 8 there are finite domains of $x_{s}$ for which the map $M$ will be continuous (w.r.t $x_{s}$ or $y_{s}$ ). Separatrix points occur when this continuity is broken.

\section{A two component model}

\subsection{Writhe of kinked field lines}

The focus in this paper is on field lines with significantly kinked $S$-shaped structure and significant aspect ratios. In order to first get an idea of the behaviour of the writhe with regards to these structures we used a two component field with

$$
k_{1 x}=3, k_{1 y}=4, \quad k_{2 x}=3, k_{2 y}=-4 .
$$

This choice gives a grid-like distribution of magnetic poles of alternating sign as depicted in (a) Figure 9. The polarity inversion lines form a square grid. This particular field was used as it allowed for $\alpha$ values which consistently produced large aspect ratio field lines with significantly kinked structure (see (b) in Figure 9 ) . This distribution maybe a little physically unrealistic, as the configuration of poles seen on the photosphere do not generally show such a degree of regularity. 


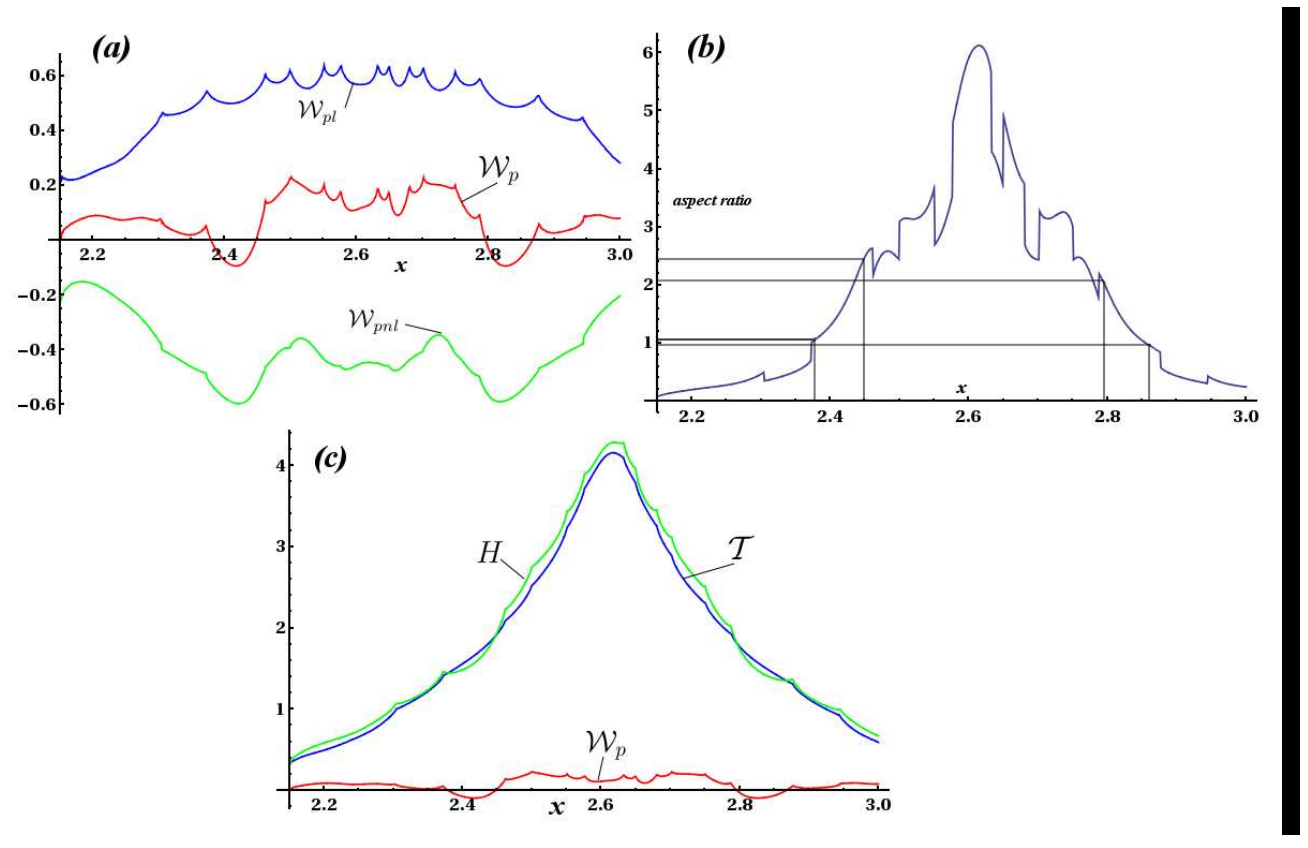

Figure 10. A representation of various quantitative characteristics of extreme $\alpha$ field line morphologies. Graphs (a)-(c) represent the measured values of field lines generated with $y=0.01 \pi, \alpha=4.9$, and varying $x$ over the set $x \in[2.1,3]$. Graph (a) depicts the $\mathcal{W}_{p}, \mathcal{W}_{p l}$ and $\mathcal{W}_{p n l}$ values. It can be seen that the majority of the $\mathcal{W}_{p}$ values are positive. We also see that the $\mathcal{W}_{p l}$ and $\mathcal{W}_{p n l}$ values are oppositely signed. The three measures do not change smoothly with changing $x$; this is a result of the field lines crossing separatrix points. Graph (b) depicts the aspect ratio of the field lines, marked are the points at which the $\mathcal{W}_{p}$ value changes sign (corresponding to the crossing points in (a)). There exists a clear peak in aspect ratio values. A high proportion are of significant value $(>1)$. Further, the discontinuous changes in aspect ratio correspond to the discontinuous changes in (a) and occur at separatrix boundaries. The helicity and $\mathcal{T}$ values are depicted in (c). The $\mathcal{T}$ dominates the helicity contribution, though we note the helicity still changes discontinuously due to the $\mathcal{W}_{p}$ contribution. In particular the negative writhing values ensure that the twist contribution is actually greater than the net helicity.

That said we shall see it produces $S$-shaped field lines comparable to more realistic distributions and gives a useful indication on the components of the $\mathcal{W}_{p}$ measure.

In practice we found that a value $\alpha=4.9$ consistently produced large aspect ratio field lines. Values for $\mathcal{W}_{p}, \mathcal{W}_{p l}$ and $\mathcal{W}_{p n l}$ of the field lines obtained by fixing $y=0.01 \pi$, and varying $x$ over the set $x \in[2.1,3]$ are shown in (see Figure $10(\mathrm{a})$ ). Similar results are found upon varying the $y$ value, that is to say the field lines generated are characteristic of the large aspect ratio field lines on this domain. Their corresponding aspect ratios are also plotted, see Figure 10 (b). The plots on graphs (a) and (b) are discontinuous at a significant number of points. The discontinuities on both graphs coincide (in terms of their $x$ values) and result from separatrix points of the field (see Section 3.3). If the separatrix boundaries are not crossed the writhing values and aspect ratio change smoothly (with 
respect to the start $x$ value), as does the field line morphology. The majority of the field lines are significantly kinked with an S-shaped structure.

We note immediately that the $\mathcal{W}_{p l}$ and $\mathcal{W}_{p n l}$ measures have opposing signs; we shall see that this is a common characteristic of the field lines studied in this note. For the majority of $x$ values $\mathcal{W}_{p}$ is positively signed as a result of $\mathcal{W}_{p l}$ being greater than $\mathcal{W}_{p n l}$. Further, the majority of their aspect ratios are significant $(>1)$. This would appear to be in opposition to the assumptions made in the introduction, which align such forward S flux ropes with negative writhing ${ }^{1}$. There are two significant start point subsets $(x \approx[2.38,2.46]$ and $x \approx[2.79,2.87])$ for which the $\mathcal{W}_{p}$ value is negative; these are also of significant aspect ratio. We shall show that these negative $\mathcal{W}_{p}$ structures are close to symmetric, though we shall also see that symmetry does not necessarily imply negative $\mathcal{W}_{p}$ values. The key aspect of the plot (a) is the opposing signs of the two writhing components. Whilst these structures would appear on viewing to have a strong degree of writhing (see for example Figure 11), the two components of the writhe almost cancel. It is the balance between the degree of local writhing and non-local writhing which decides the sign of the writhing.

The plot labelled (c) (Figure 11) shows the $\mathcal{W}_{p}, \mathcal{T}$ and $H$ values for the field lines. It is clear that the twist $\mathcal{T}$ is the dominant helicity contribution in all cases. Partly this twisting dominance is a result of the opposing local and non-local writhing components of the net writhing measure. What is of interest here is that the possibility of negative writhing values leads to an increase in the twisting; indeed these are the only cases for which the twist is greater than the helicity. So this result would oppose the notion that an increase in writhing always leads to a decrease in the magnitude of the twisting of the field line or flux rope axis.

Figure 11 depicts field lines structures $\mathbf{B}$ in two forms. These configurations represent the field line morphologies over a range of $x$ values for which the $\mathcal{W}_{p}$ measure changes sign from positive to negative and then back. As $x$ is increased we see two geometrical changes in field line configuration. First of all the height of the field line increases and secondly the section of field line around the apex appears to rotate towards the viewer in an clockwise direction. In Figure 11 (b) we see this rotation appears to coincide with a change from asymmetry with bias towards the upward pointing section of the field line, through symmetric configurations and then onto an asymmetric configuration, whose bias is towards the downward pointing section of the field line. This changing morphology occurs smoothly with $x$, but we have only depicted these four values for clarity.

The analysis of the above paragraph would appear to suggest that the $\mathcal{W}_{p}$ value will be negative for field lines whose morphologies are symmetric and of significant scale. As previous simulations have tended to use symmetric flux ropes and field lines, this could at first sight validate the assumptions made in the introduction.

However, we now explore the consequences of crossing a separatrix point. Diagrams (a) and (b) in Figure 12 depict the field lines drawn out for $\alpha=4.9$, $y=0.01 \pi$ and $x=2.47$. We see in plot (a) Figure 10 this configuration occurs

\footnotetext{
${ }^{1}$ A large, equally spaced (in terms of the start $x$ value), sample of field line configurations were
} plotted and all were found to be $S$-shaped. 

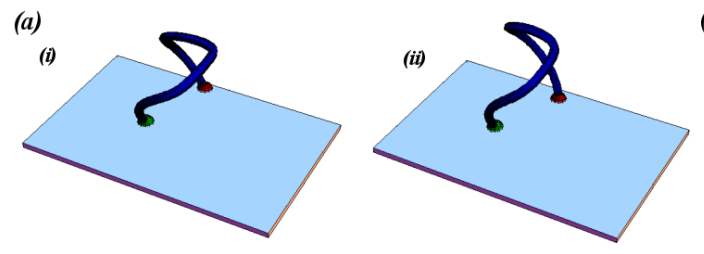

(b)
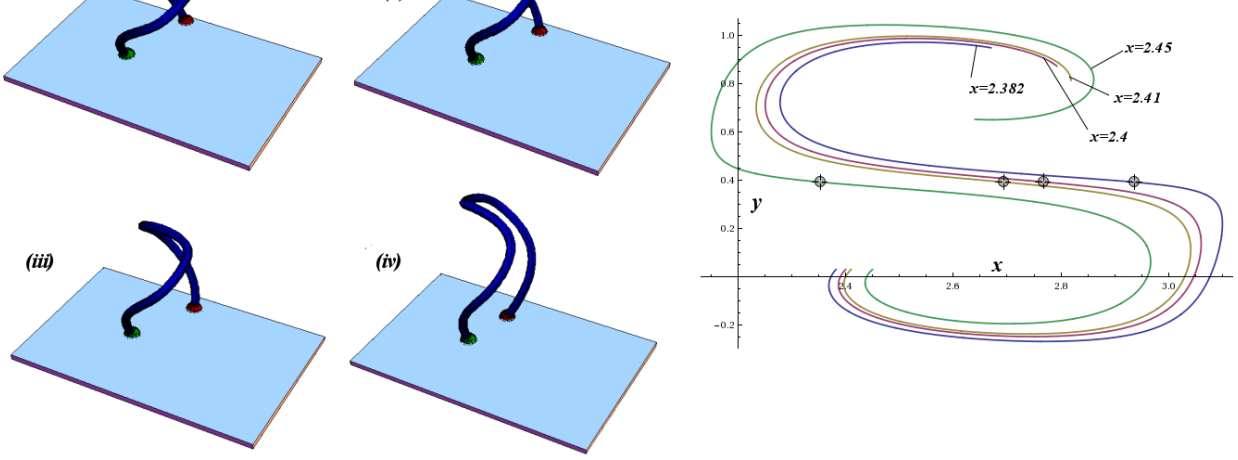

Figure 11. Representative field line configurations of both positive and negative $\mathcal{W}_{p}$ values. In (a), (i) to (iv) represent the field line configurations $\mathbf{B}$, obtained for $\alpha=4.9, y=0.01 \pi$ and $x$ values of $x=2.379$ (i), $x=2.4$ (ii), $x=2.41$ (iii) and $x=2.45$ (iv). The field lines show the apex section of the configurations rotating towards the viewer, in a clockwise direction. The $\mathcal{W}_{p}$ values are 0.00238213 (i), -0.0725992 (ii), -0.0895121 (iii) and 0.0181881 (iv). During this rotation the sign of $\mathcal{W}_{p}$ changes from positive to negative and then back to positive. We also note an increase in apex height across (i) to (iv). Graph (b) depicts these field lines projected onto the $x-y$ plane, the cross hair indicates the field's apex. The configuration at $x=2.379$ is significantly asymmetric with a greater degree of total curvature in the section of curve joining the start point to the apex. As $x$ is increased we see, in the $x=2.40$ and $x=2.41$ configurations, a decrease in asymmetry. Finally the $x=2.45$ sigmoid shape is again significantly asymmetric with the greater degree of curvature in the endpoint-apex section of the field line configuration. There has been a transfer of asymmetry from the upward moving section of $\mathbf{B}$ to the downward moving section.

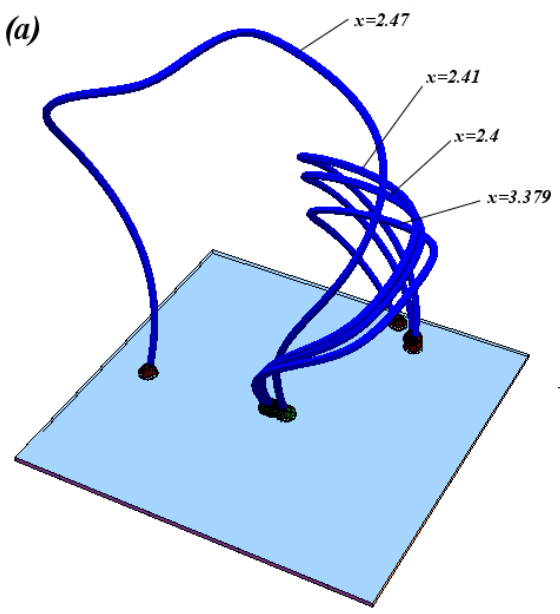

(b)

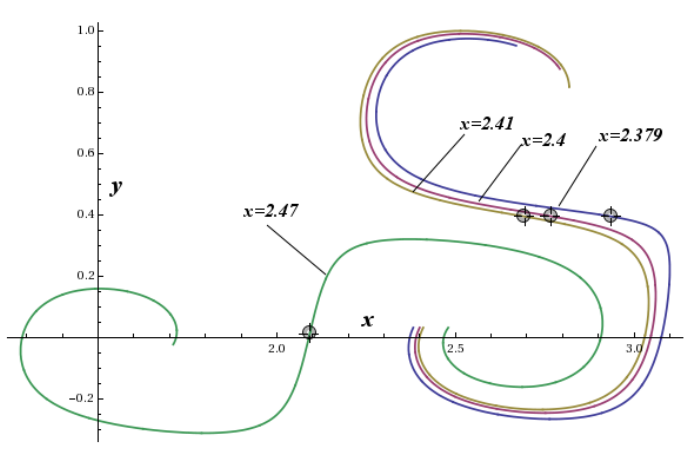

Figure 12. Illustrating field lines on either side of a separatrix point. Depicted are the field line configurations $\mathbf{B}$ (a) and their projections (b); obtained for $\alpha=4.9, y=0.01 \pi$ and $x$ values of $x=2.379, x=2.40, x=2.41$ and $x=2.47$. The first three configurations $(x=2.379$, $x=2.40, x=2.41$ ) have already been discussed in Figure 11. The $x=2.47$ configuration has a $\mathcal{W}_{p}$ value of 0.138 . The final field line has a $x$ value which belongs to a neighbouring behavioural mode. In (b) we see this configuration seems to be close to symmetric in structure. 


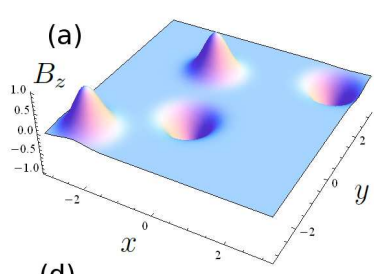

(d)

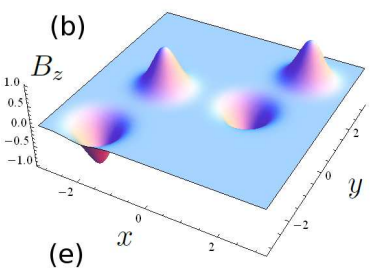

(e)

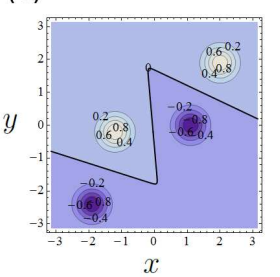

(c)

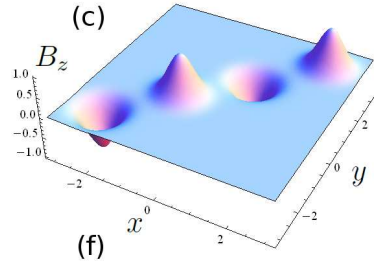

(f)

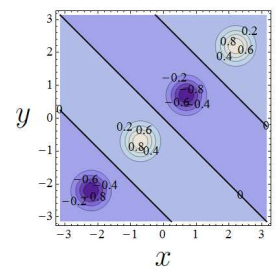

Figure 13. Depictions of the $B_{z}(x, y, z=0)$ functions used to specify field lines resulting from quadrupole distributions with 2 positive and 2 negative poles. Figures (a),(b), and (c) plot the $B_{z}$ component as a function of $x$ and $y,(\mathrm{~d}),(\mathrm{e})$ and (f) are the corresponding contour plots with the polarity inversion line depicted as a thick black line. Figure (c) depicts an "EBR" type configuration (using the classification in MGY), it has three polarity inversion lines (f). Figure (b) depicts an I/EBR type configuration. It has an S-shaped polarity inversion line. The distribution in (a) is a less ordered set of poles. It has a Z-shaped polarity inversion line.

to the right (its $x$ value is larger) of a discontinuous jump in the various writhe values, i.e. it crosses as separatrix point. This field appears to be symmetric, with a positive $\mathcal{W}_{p}$ value and has a significantly greater height than the lines diverging the other side of the separatrix point. The negative $\mathcal{W}_{p}$ values may be indicative of a particular behavioural mode rather than a function of the field line symmetry. Observation of the field lines (and their projections) over the $x$ range covered in Figure 10 did not suggest any consistent relationship between the field line's symmetry and it's writhing value.

\section{More realistic $B_{z}$ distributions}

The observations of Mackay, Gaizauskas, and Yeates (2008) (MGY) suggest that a large proportion of solar filaments are formed in regions of the photosphere in which the vertical component of the field $B_{z}$ has four distinct poles, 2 positive and 2 negative. Following the classification scheme suggested in MGY one set, termed EBR Filaments, have the poles approximately lined up with alternating signs (c.f (f) in Figure 13 with Figure 2 (b) of MGY). A second set, termed I/EBR type filaments have alternate signs with an offset (c.f (e) in Figure 13 with Figure 2 (c) of MGY). In this section we define a function $B_{z}(x, y)$ function which mimics these distributions and apply the polar writhe measure to field lines whose aspect ratio is greater than 1. In addition we consider a third 4pole distribution with a more asymmetric scattering (Depicted in (a) and (d) of Figure 13). 
The fields were created using Gaussian poles, defined as

$$
g p\left(x, y, d, \sigma, x_{c}, y_{c}\right)=d \mathrm{e}^{-\sigma\left(\left(x-x_{c}\right)^{2}+(y-y c)^{2}\right)} .
$$

Here $d$ is the maximum density, $\sigma$ controls the rate of decay and $\left(x_{c}, y_{c}\right)$ the Cartesian location of the pole's centre. The distributions were specified by choosing these parameters for each of the four poles. The poles were chosen to lie in a domain $x \in[-\pi, \pi], y \in[-\pi, \pi]$ and the expression was expanded as a Fourier series on this domain to include all coefficients $\left(k_{x}=i, k_{y}=j\right), i, j \in 0,1,2,3,4,5$, (excluding the component $k_{x}=k_{y}=0$ ). For these fields we require $\alpha<1$ (see Section 3.2). In general there were only certain sub domains of $\left(x_{c}, y_{c}\right)$ on which field lines of the required aspect ratio were generated.

During this investigation the authors did evaluate the relevant helicity measures for aspect ratio field lines $<1$. The range of behaviour appears to be much more variable with regards to their various inter-relationships (though the vast majority had positive helicity for positve $\alpha$ ) and a decision was made to concentrate on the large aspect ratio field lines for this study.

5.1. Results - case (a)

We consider the distribution

$$
\begin{aligned}
B_{z}(x, y)= & g p(x, y, 1,4.2,-2.3,-2.4)+g p(x, y,-1,4.2,-0.3,-0.9) \\
& +g p(x, y, 1,4.2,-0.3,1.7)+g p(x, y,-1,4.2,2.4,-2.2),
\end{aligned}
$$

depicted in (a) and (d) of Figure 13. A value of $\alpha=0.97$ was chosen so that a higher proportion of kinked field lines would be generated. A grid of sampling $(x, y)$ values was used to search for start point domains for which field lines had a significant aspect ratio and kinked field structure. Two particular regions were found to consistently produce such lines. From these regions two sets $x_{s}=$ $-3 / 5, y_{s} \in[1.5,2.2]$ and $x_{s}=-2.1, y_{s} \in[-2.68,-3.1]$ were studied (similar results would be found for $x_{s}$ values in a neighbourhood $x_{s}=-3 / 5$ and $x_{s}=$ -2.1 , i.e. the following results are characteristic of the regions).

In Figure 14 (a)-(d) we have plotted the various field line measures of interest on the set $x_{s}=-3 / 5, y_{s} \in[1.5,2.2]$. The conclusions are similar to those of Section 4 . The helicity is dominated by its twist component (b), $\mathcal{W}_{p}$ has both positive and negative values and its components $\mathcal{W}_{p l}$ and $\mathcal{W}_{p n l}$ are of opposing sign (a). Finally, we note the field lines with negative $\mathcal{W}_{p}$ have aspect ratio $>1$. The discontinuities in the plots correspond to separatrix boundaries. In (d) we have plotted projections of a selection of field lines whose aspect ratio is greater than 1 . They all have a pronounced S-shape and belong to one separatrix line (d).

From the data shown in Figure 15 for the set $x_{s}=-2.1, y_{s} \in[-2.68,-3.1]$ we can draw similar conclusions i.e. for large aspect ratio field lines $(>1)$ the sign of $\mathcal{W}_{p}$ is determined by the relative values of $\mathcal{W}_{p l}$ and $\mathcal{W}_{p n l}$, which have opposing signs (a), and the helicity is dominated by its $\mathcal{T}$ component (b). We note the only field lines for which the writhe helicity is comparable to its twist component is 
(a)

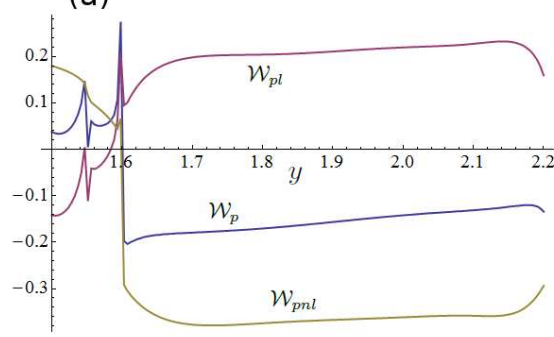

(c)

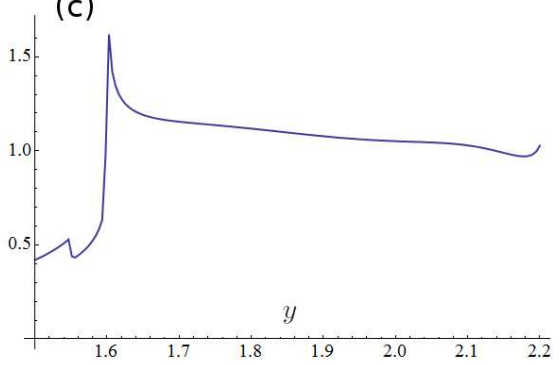

(b)

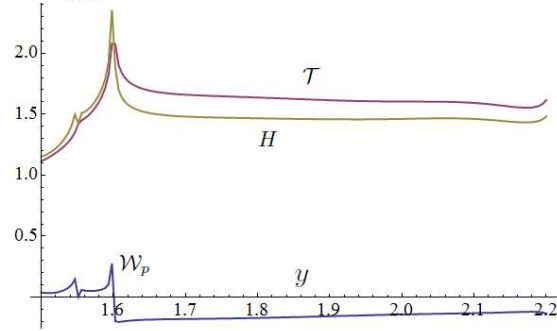

(d)

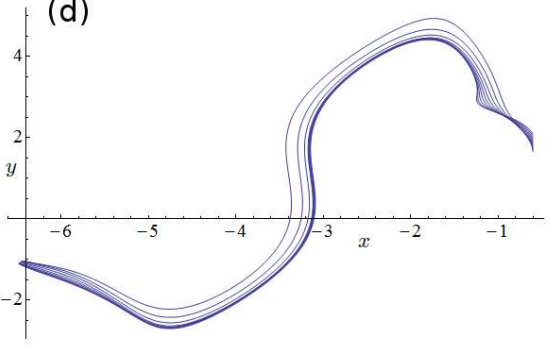

Figure 14. Plots of various characteristic measures of field lines produced using (19) and start points form $x_{s}=-3 / 5, y_{s} \in[1.5,2.2]$. We see in (a) $\mathcal{W}_{p}$ has both positive and negative values (mainly negative) and that $\mathcal{W}_{p n l}$ and $\mathcal{W}_{p l}$ are generally opposite in sign. In (b) we see that the Helicity $H$ is dominated by its $\mathcal{T}$ component for field lines of aspect ratio $>1$ (c). In both cases the sharp changes in the graphs correspond to changes in separatrix surface. Figure (d) is a projection of a selection of the field lines whose aspect ratio is greater than one onto the $\mathrm{x}-\mathrm{y}$ plane. They all have a pronounced S-shape.

for field lines with small aspect ratios (c). Again, the discontinuities correspond to separatrix boundaries. We see in (d) that two sets of the field lines have a pronounced $S$ shape however there are two subsets which could be interpreted as having $Z$-shapes. We see a depiction of two examples of such fields in Figure 16. The field line depicted in (a) and (c) has $\left(x_{s}=-2.1, y_{s}-2.764\right)$, aspect ratio $\approx 1.745$, and has helicity measures $\mathcal{W}_{p}=0149, \mathcal{W}_{p l}=0.086, \mathcal{W}_{p n l}=0.063$, $\mathcal{T}=2.807$ and $H=2.957$ (all to 3.d.p). The field line depicted in (b) and (d) has $\left(x_{s}=-2.1, y_{s}=-3.079\right)$, aspect ratio $\approx 0.376$ (below the ratio we are generally interested in here). It has helicity measures $\mathcal{W}_{p}=0.125, \mathcal{W}_{p l}=0.070$, $\mathcal{W}_{p n l}=0.055, \mathcal{T}=1.311$ and $H=1.436$. Both field lines have only one turning point in the $\hat{z}$ direction (i.e. they are not dipped). Also we note both have $\mathcal{W}_{p l}$ and $\mathcal{W}_{p n l}$ values of the same sign. Such morphologies raise the possibility of mistakenly classifying such field lines as having negative helicity when in fact the helicity matches its $\alpha$ value.

5.2. Results - case (b)

We now consider the distribution

$$
B_{z}(x, y)=g p(x, y, 1,4.2,-1.9,-2.4)+g p(x, y,-1,4.2,-1.1,0)
$$


(a)

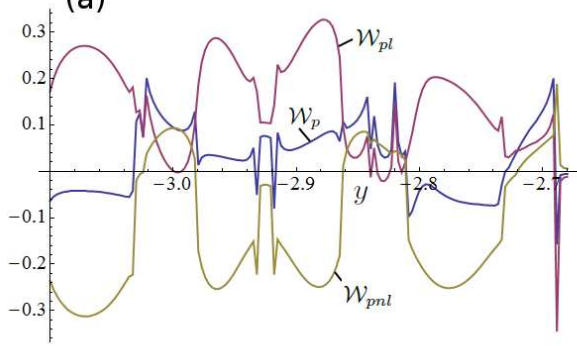

(c)

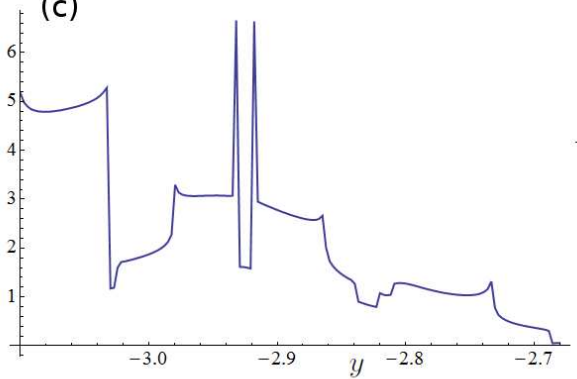

(b)

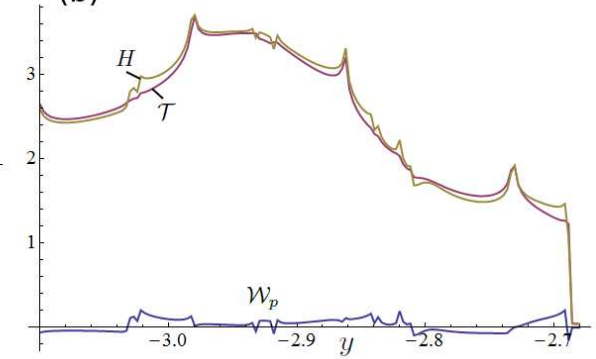

(d)

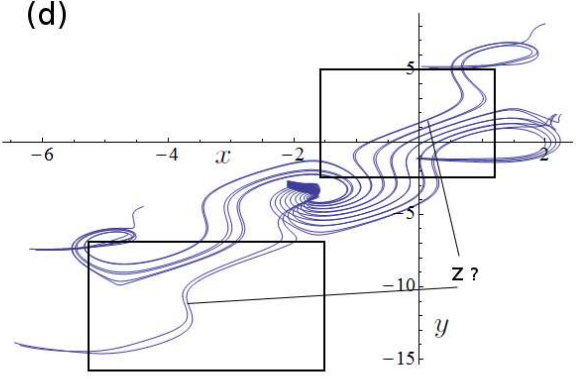

Figure 15. Plots of various characteristic measures of field lines produced using (19) and start points form $x_{s}=-2.1, y_{s} \in[-2.68,-3.1]$. Once again we see in (a) $\mathcal{W}_{p}$ exhibiting positive and negative values and $\mathcal{W}_{p l}$ and $\mathcal{W}_{p n l}$ often having opposing signs, though not always. In (b) we see in all but a small region at the higher end of the $y$ scale that the helicity is dominated by its twist component. In (c) we see the region in which $\mathcal{W}_{p}$ is comparable to $\mathcal{T}$ corresponds to field lines of small aspect ratio. In $(\mathrm{d})$ we see the field line projections onto the $x-y$ plane. There are two sets of field lines with pronounced S-shapes. the two sets of field lines marked $\mathrm{Z}$ ? on the diagram could be interpreted as having a Z-shaped structure.

$$
+g p(x, y, 1,4.2,0.2,1)+g p(x, y,-1,4.2,1.9,2.4)
$$

depicted in (b) and (e) of Figure 13. Again a value of $\alpha=0.97$ was used. As in the previous section a gird of start points was used, though only one consistent region of start point parameters was found to lead to significantly kinked field lines of aspect ratio $>1$. From this domain the set $x_{s}=-0.5, y_{s} \in[-0.1,0.4]$ was chosen.

In Figure 17 we see the behaviour of the various measures on the set $x_{s}=$ $-0.5, y_{s} \in[-0.1,0.4]$. For most of the domain $\mathcal{W}_{p l}$ and $\mathcal{W}_{p n l}$ have opposing signs, though there are $y$ domains for which $\mathcal{W}_{p l}$ and $\mathcal{W}_{p n l}$ have the same sign. The twist component dominates the helicity of the local flux bundle. The larger difference between $\mathcal{W}_{p}$ and $\mathcal{T}$ corresponds to the field lines with larger aspect ratios (c). In (d) we see the field line projections do not appear to have a significant S-shaped morphology. We plot some three dimensional examples of these field lines in Figure 18. Figures (e)-(f) correspond to the field lines (a)-(d) as viewed from above. Their start points are $(-1.5,0.2)(\mathrm{a}),(-1.5,0.25)(\mathrm{b})$, $(-1.5,0.3)(\mathrm{c})$ and $(-1.5,0.35)$. Their respective helicity measures are listed in Table 1. 

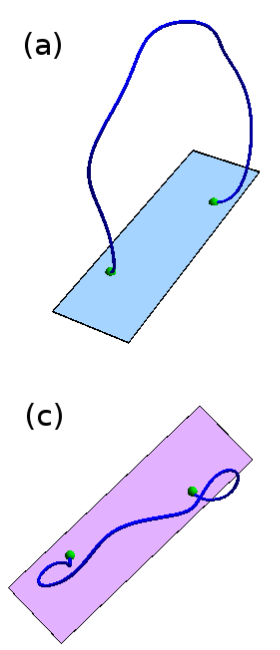

(b)

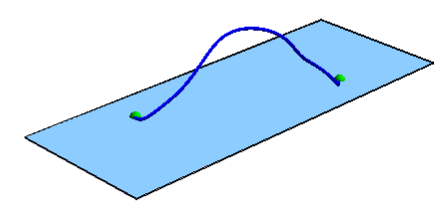

(d)

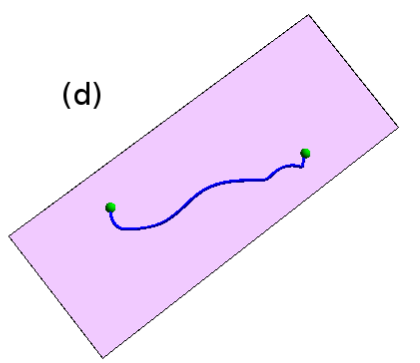

Figure 16. Depictions of field line structures which could be viewed as Z-shaped. Figures (c) and (d) are the respective views of (a) and (b) from directly above (i.e. along $-\hat{z}$ ). The central section of both lines could be viewed as $Z$ shaped. Both field lines have positive $\alpha$ values usually associated with $\mathrm{S}$ shapes.

Table 1. Helicity measures of field lines displayed in Figure 18.

\begin{tabular}{ccccccc} 
Field & $\mathcal{W}_{p}$ & $\mathcal{W}_{p l}$ & $\mathcal{W}_{p n l}$ & $\mathcal{T}$ & $H$ & Aspect ratio \\
\hline (a) & 0.214 & 0.329 & -0.116 & 4.205 & 4.418 & 3.293 \\
(b) & 0.352 & 0.378 & -0.026 & 6.037 & 6.391 & 2.748 \\
(c) & 0.163 & 0.102 & 0.061 & 3.008 & 3.171 & 1.317 \\
(d) & 0.178 & 0.120 & 0.058 & 2.570 & 2.749 & 1.356.
\end{tabular}

All fields have positive $\mathcal{W}_{p}$ and their $\mathcal{T}$ values are much larger than their $\mathcal{W}_{p}$ measure. Two have positive $\mathcal{W}_{p n l}$ values. From Figures (e)-(h) in Figure 18 we see their mid sections have a $Z$ shaped structure. Only (f) has a noticeably $S$-shaped global structure. Despite this their $\mathcal{W}_{p}$ values are comparable to the more clearly $S$-shaped structures shown in (d) Figure 14. This analysis offers further evidence that estimating the writhing of flux ropes and field lines purely from line of sight observation could lead to significant error without a specific knowledge of the field line's full three dimensional structure.

\subsection{Results case (c)}

We now consider the distribution of poles given by

$$
\begin{array}{r}
B_{z}(x, y)=g p(x, y,-1,4.2,-2.2,-2.2)+g p(x, y, 1,4.2,-0.7,-0.7) \\
+g p(x, y,-1,4.2,0.7,0.7)+g p(x, y, 1,4.2,2.2,2.2),
\end{array}
$$



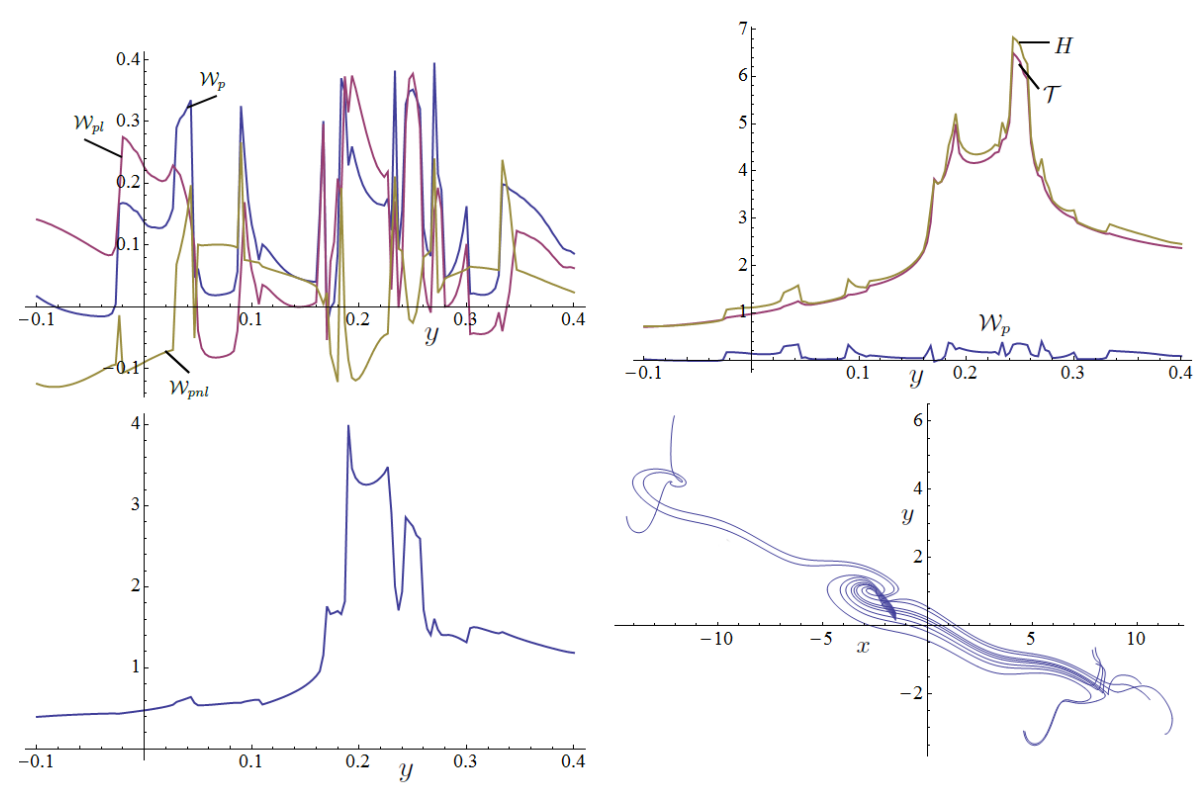

Figure 17. Plots of various characteristic measures of field lines produced using (20) and start points form $x_{s}=-0.5, y_{s} \in[-0.1,0.4]$. In (a) we see the writhe measures show significant changes on crossing separatrix boundaries. For the majority of the domain $\mathcal{W}_{p}$ is positive and $\mathcal{W}_{p l}$ and $\mathcal{W}_{p n l}$ are opposite in sign, though there are $y$-ranges for which they have the same sign. In (b) we see the $\mathcal{T}$ dominates the helicity of the field line for field lines whose aspect ratio are $>1$ (c). Figure (d) depicts projections of the field lines onto the $x-y$ plane. The field lines do not appear to have significantly S-shaped structure and could be interpreted as being $Z$-shaped on its interior.

as depicted in (c) and (f) of Figure 13. A value of $\alpha=0.97$ was used. A grid of start points was used to identify two sets of parameter values which produced significantly kinked field lines. From these domains the sets $x_{s}=2.7, y_{s} \in[1.8,2.7]$ and $x_{s}=2.1, y_{s} \in[2.4,2.48]$ were selected.

In Figure 19 we see the behaviour of the various measures on the set $x_{s}=$ $2.7, y_{s} \in[1.8,2.7]$. Once again we see the $\mathcal{W}_{p l}$ and $\mathcal{W}_{p n l}$ measures are generally of opposing sign (a). For most of the domain $\mathcal{W}_{p}$ is positive but it can take on negative values. In (b) and (c) we see that the helicity is dominated by its twist component for field lines of significant aspect ratio $(>1)$. We note that the values of the aspect ratios are significantly high for these tall field lines. They have a pronounced $S$-shape and their field lines measures have similar behaviour to those examples studied in Sections $4,5.1$ and the set $x_{s}=2.1, y_{s} \in[2.4,2.48]$ which we now study.

In Figure 20 we see the behaviour of the various measures on the set $x_{s}=$ $2.1, y_{S} \in[2.4,2.48]$. This domain is quite small and only covers one separatrix line. The $\mathcal{W}_{p l}$ and $\mathcal{W}_{p n l}$ measures are of opposing sign and their morphology is of significantly kinked $S$-shapes. The writhe measure generally has a positive value but can take on negative values. In (b) we see that once again the helicity has 


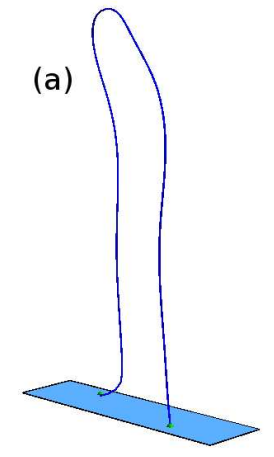

(e)

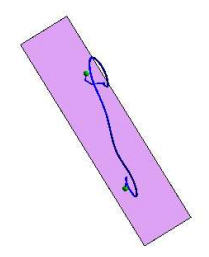

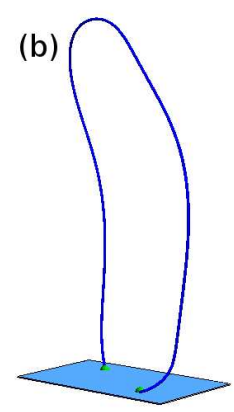

(f)

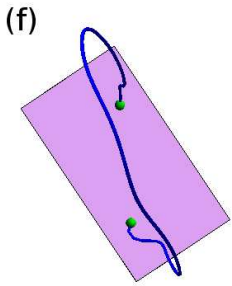

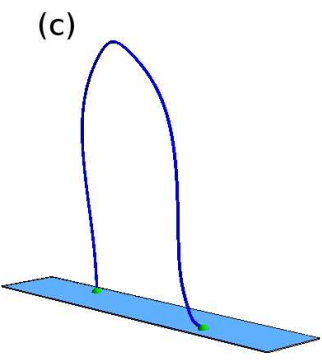

(d)

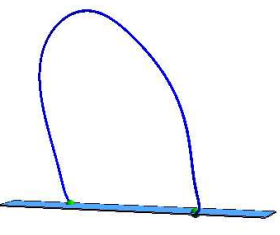

(g)

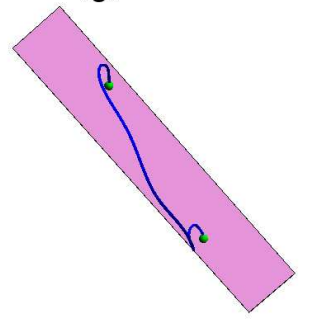

(h)

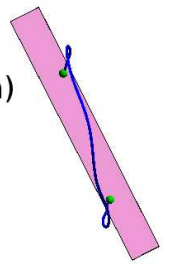

Figure 18. Some depictions of the type of field lines whose characteristics are evaluated in Figure 17. Figures (e)-(h) correspond to views of (a)-(d) from above, such as one might obtain from line of sight imaging. It is only really field (f) which has noticeably $S$ shaped characteristics. The other fields could be said to have $Z$-shaped characteristics.

the same sign as its twist contribution. Though the $\mathcal{W}_{p}$ measure can contribute a significant proportion of the helicity (approximately a third at its highest value).

\section{Summary and conclusions}

We have evaluated a range of field line morphologies using a simple linear forcefree model. In particular we have demonstrated the existence of the following possibilities for the signs of helicity, twist, and writhe:

- For taller or larger field line structures (aspect ratio $>1$ and positive $\alpha$ ) all field lines examples had positive helicity which agreed with their $\alpha$ value. The twist component of the helicity was always larger than the writhe helicity, and often the dominant component. For significantly kinked S-shaped field lines this is a result of the local and non-local writhing measures being of opposite sign, leading to small net writhing values. The writhe measure of such field lines could was seen to take on both positive and negative values. Generally the $\mathcal{W}_{p l}$ and $\mathcal{W}_{p n l}$ measures had opposing signs and their relative values determined the sign of $\mathcal{W}_{p}$. This allows for the possibility that, for S-shaped positive helicity structures, an increase in absolute writhing could lead to both increasing and decreasing in the magnitude of twisting. See Sections 4 and 5 . 
(a)

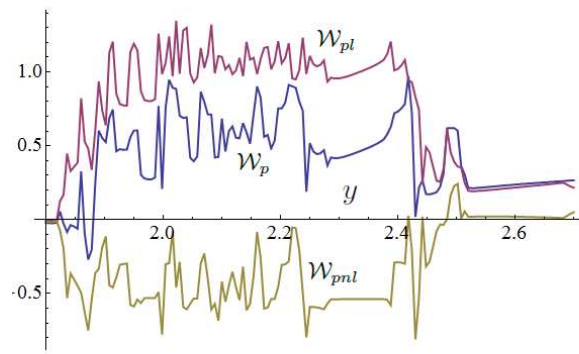

(c)

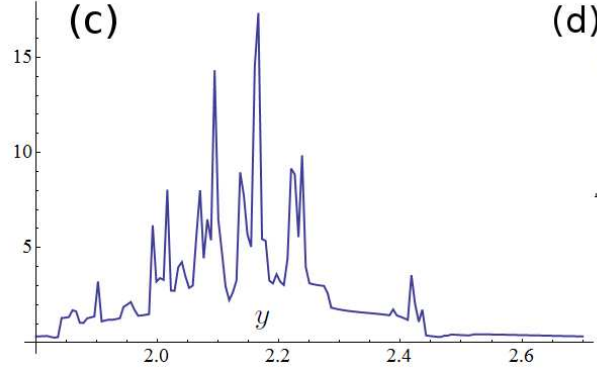

(d) (b)
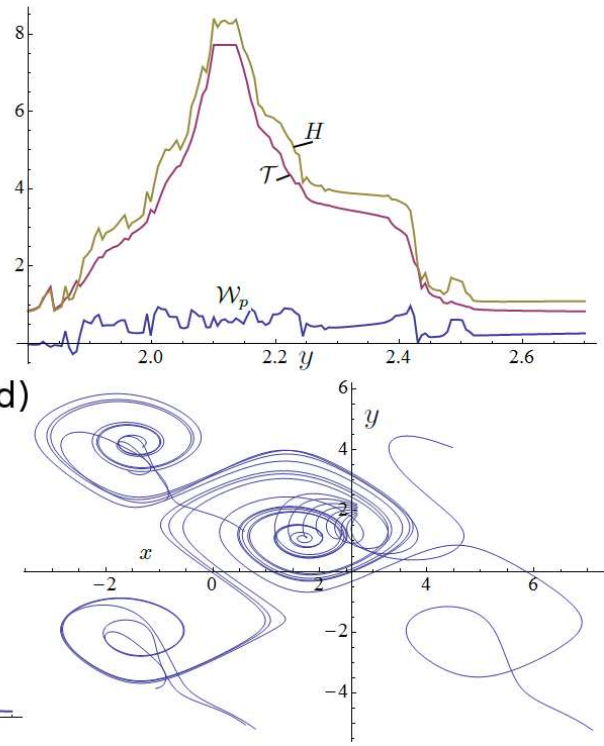

Figure 19. Plots of various characteristic measures of field lines produced using (21) and start points form $x_{s}=2.7, y_{s} \in[1.8,2.7]$. In (a) we see the $\mathcal{W}_{p l}$ and $\mathcal{W}_{p n l}$ are generally of opposing sign. In (b) and (c) we see the helicity is dominated by its twist components for field lines of significant aspect ratio. In (d) we observe that the field lines have significantly kinked S-shaped characteristics.

- Field lines of aspect ratio $>1$ and positive $\alpha$ were found which could be interpreted as being $\mathrm{Z}$ shaped (at least around their central region). These structures all had positive helicity values and their $z$-component had a single turning point. Their writhing values were comparable in magnitude to field lines of significant $S$-shape and had both positive and negative writhe values. See Section 5

- The differing behavioural modes, separated by separatrix points (see Section 3.3 for a definition), were shown to determine the particular morphological characteristics of the field line (i.e. its helicity or writhing values). Simple observations of the degree symmetry or asymmetry, or aspect ratio of the field line were not seen to be consistent indicators of the value of the field lines helicity and writhing values. This would suggest that simple qualitative or quantitative observations of two dimensional images could lead to incorrect evaluations of the field line helicity.

\subsection{Conclusions}

The results contained within this note offer a glimpse of a subtle relationship between quantitative helicity measures of flux tubes and their observed morphology. The major conclusion is that the writhe helicity of field lines and flux ropes cannot be accurately evaluated without full knowledge of the flux rope's central 

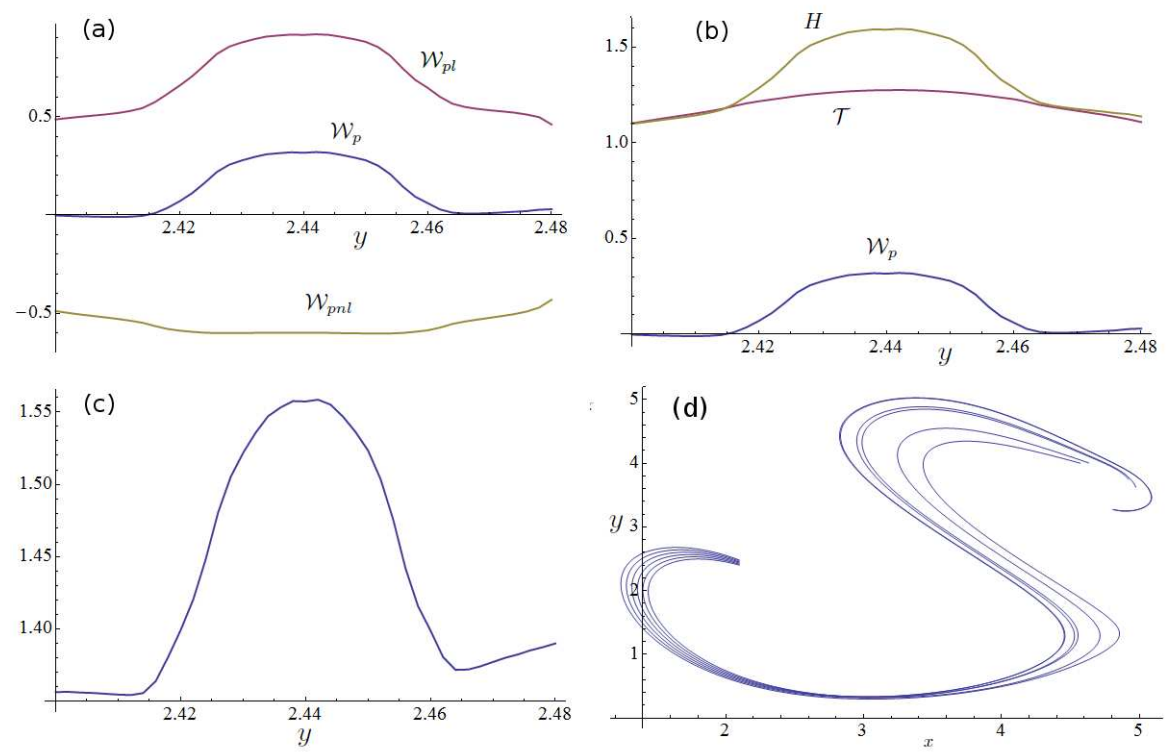

Figure 20. Plots of various characteristic measures of field lines produced using (21) and start points form $x_{s}=2.1, y_{s} \in[2.4,2.48]$. This smaller regions is confined to a single separatrix layer. In (a), the $\mathcal{W}_{p l}$ and $\mathcal{W}_{p n l}$ are of opposing sign and $\mathcal{W}_{p}$ can be both positive and negative. In (b), the twist is again larger than the writhe. In (c) we see these field lines have a small range of aspect ratios. In (d) we see the field lines have a pronounced S-shape.

axis geometry. However, the models used were comparatively simple compared to the wide range of field modelling techniques in solar literature. The aim was to demonstrate that one cannot necessarily infer the properties of sigmoidal flux ropes and field lines by a simple visual measurement, rather than comment on any current coronal active region field models.

Acknowledgement Chris prior wishes to thanks John Brooke for his comments and observations on the manuscript. This paper is based on work supported by both the EPSRC and Award No. KUK-C1-013-04 made by King Abdullah University of Science and Technology (KAUST).

\section{References}

Berger, M.A.: 1984,. Geophysical and Astrophysical Fluid Dynamics 30, 79.

Berger, M.A., Field, G.B.: 1984,. Physics of Fluids 47, 133.

Berger, M.A., Prior, C.B.: 2006,. J. Physics A: Mathematical and General 39, 8321.

Călugăreanu, G.: 1959,. Rev. Math. Pure. Appl. 4, 5.

Călugăreanu, G.: 1961,. Comm. Acad R.P. Romie 11, 588.

Fan, Y., Gibson, S.E.: 2004,. Astrophysical J. 609(2), 1123.

Gary, G.A.: 1989,. Astrophysical J. Supplement 69, 323.

Green, L.M., Kliem, B., Török, T., van Driel-Gezstelyi, L., Attrillm, G.D.R.: 2007,. Solar Physics 246(2), 365 .

Handy, B.N., et al.: 1999,. Solar Physics 187(2), 229.

Heyvaerts, J., Priest, E.R.: 1984,. Astronomy and Astrophysics 137, 63. 
Kaiser, M.: 2005,. Adv. Space Resarch 36(8), 1483.

Kliem, B., Titov, V.S., Török, T.: 2004,. Astronomy and Astrophysics 413, 23.

Kosugi, T., et al.: 2007,. Solar Physics 243(1), 3.

Liu, R., Gilbert, H.R., Alexander, D., Su, Y.: 2008,. Astrophysical J. 680(2), 1508.

Longcope, D.: 2005,. Living Reviews of Solar Physics 2(7), ?.

Low, B.C., Berger, M.A.: 2003,. Astrophysical J. 580(1), 644.

Mackay, D.H., Gaizauskas, V., Yeates, A.R.: 2008,. Astrophysical J. 248, 51.

Mackay, D.H., Gaizauskas, V., Rickard, G.J., Priest, E.R.: 1997,. Solar Physics 486, 534.

Magara, T., Longcope, D.: 2001,. Astrophysical J. 559, 55.

Magara, T., Longcope, D.: 2003,. Astrophysical J. 586, 630.

Morse, P.M., Feshbach, H.: 1953, New york: Mcgraw hill.

Muglach, K., Wang, Y.M., Kliem, B.: 2009,. Astrophysical J. 703, 976

Nakagawa, Y., Radius, M.A., Billings, D.E., Catamaran, D.: 1971,. Astrophysical J. 19(1), 72 .

Pevstov, A.A.: 2008,. Astrophysical J. 29(1-2), 49.

Priest, E.R.: 1981, Kluwer academic pub: Dordrecht, holland.

Rust, D.M.: 2003,. Adv. Space. Res. 32(10), 1985.

Rust, D.M., Kumar, A.: 1996,. Astrophysical J. 464(2), 199.

Tian, L., Liu, Y., Yang, J., Alexander, D.: 2005,. Solar Physics 229(1), 63.

Titov, V.S., Demoulin, P.: 1999,. Astronomy and Astrophysics 351, 707.

Török, T., Kliem, B.: 2005,. Astrophysical J. 630(1), 97.

Török, T., Berger, M.A., Kliem, B.: 2010,. Astronomy and Astrophysics 516(49), 49.

Török, T., Kliem, B., Titov, V.S.: 2004,. Astronomy and Astrophysics 413, 27.

Tsuneta, A., et al.: 1991,. Solar Physics 136(1), 37. 
SOLA: forcefreewrithe1.tex; 31 October 2011; 9:21; p. 26 



\section{RECENT REPORTS}

43/11 Coupling fluid and solute dynamics within the ocular surface tear Zubkov film: a modelling study of black Line osmolarity

Breward

Gaffney

44/11 A prototypical model for tensional wrinkling in thin sheets

Davidovitch

Schroll

Vella

Adda-Bedia

Cerda

45/11 A fibrocontractive mechanochemical model of dermal wound closure incorporating realistic growth factor

Murphy

Hall

Maini

McCue

McElwain

46/11 A two-compartment mechanochemical model of the roles of trans-

Murphy

forming growth factor $\beta$ and tissue tension in dermal wound heal-

Hall ing

Maini

McCue

McElwain

47/11 Effects of demographic noise on the synchronization of a metapopulation in a fluctuating environment

Lai

Newby

Bressloff

48/11 High order weak methods for stochastic differential equations based on modified equations

Abdulle

Cohen

Vilmart

Zygalakis

49/11 The kinetics of ice-lens growth in porous media

Style

Peppin

50/11 Wound healing angiogenesis: the clinical implications of a simple

Flegg mathematical model

Byrne

Flegg

McElwain

51/11 Wound healing angiogenesis: the clinical implications of a simple

$\mathrm{Du}$ mathematical model

Gunzburger

Lehoucq

Zhou

52/11 Image Inpainting based on coherence transport with Adapted dis-

März tance functions

53/11 Surface growth kinematics via local curve evolution

Moulton

Goriely

54/11 A multiple scales approach to evaporation induced Marangoni convection

Hennessey

Münch

55/11 The dynamics of bistable liquid crystal wells

Luo

Majumdar

Erban

56/11 Real-Time Fluid Effects on Surfaces using the Closest Point Auer Method 
58/11 Riemann-Cartan Geometry of Nonlinear Dislocation Mechanics Yavari

Goriely

59/11 Helices through 3 or 4 points? Goriely

Neukirch

Hausrath

60/11 Bayesian data assimilation in shape registration Cotter

Cotter

Vialard

61/11 Asymptotic solution of a model for bilayer organic diodes and Richardson solar cells

Please

Kirkpatrick

62/11 Neural field model of binocular rivalry waves

Bressloff

Webber

63/11 Front propagation in stochastic neural fields

Bressloff

Webber

64/11 Stability estimates for a twisted rod under terminal loads: a threedimensional study

Majumdar

Prior

Goriely

65/11 Adaptive Finite Element Method Assisted by Stochastic Simula-

Cotter tion of Chemical Systems

Vejchodsky

Erban

Copies of these, and any other OCCAM reports can be obtained from:

Oxford Centre for Collaborative Applied Mathematics

Mathematical Institute

24 - 29 St Giles'

Oxford

OX1 3LB

England

www.maths.ox.ac.uk/occam 